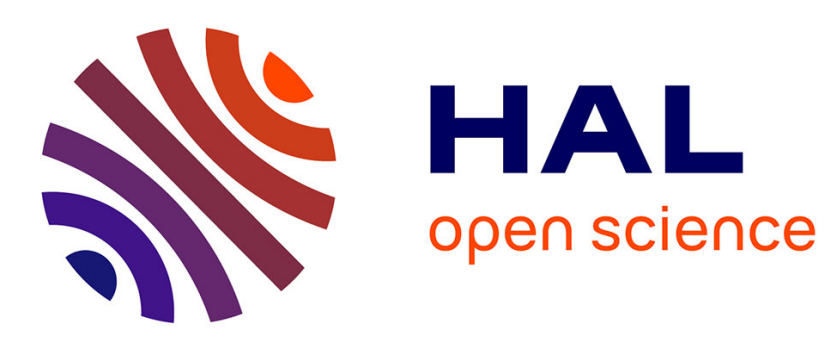

\title{
On the Dynamic Properties and Optimum Control of Parallel Manipulators in the Presence of Singularity
}

Sébastien Briot, Vigen Arakelian

\section{To cite this version:}

Sébastien Briot, Vigen Arakelian. On the Dynamic Properties and Optimum Control of Parallel Manipulators in the Presence of Singularity. 2008 IEEE International Conference on Robotics and Automation (ICRA 2008), May 2008, Pasadena, United States. hal-00451915

\section{HAL Id: hal-00451915 \\ https://hal.science/hal-00451915}

Submitted on 25 Jun 2019

HAL is a multi-disciplinary open access archive for the deposit and dissemination of scientific research documents, whether they are published or not. The documents may come from teaching and research institutions in France or abroad, or from public or private research centers.
L'archive ouverte pluridisciplinaire HAL, est destinée au dépôt et à la diffusion de documents scientifiques de niveau recherche, publiés ou non, émanant des établissements d'enseignement et de recherche français ou étrangers, des laboratoires publics ou privés. 


\title{
On the Dynamic Properties and Optimum Control of Parallel Manipulators in the Presence of Singularity
}

\author{
Sébastien Briot and Vigen Arakelian
}

\begin{abstract}
It is known that a parallel manipulator with a singular configuration can gain one or more degrees of freedom and become uncontrollable. That is it might not reproduce a stable motion under a prescribed trajectory. However, it is proved experimentally that there is possible passing through the singular zones. This was simulated and shown on numerical examples and illustrated on several parallel structures. In this paper, we determine the optimal dynamic conditions generating a stable motion inside the singular zones. The obtained results show that the general condition for passing through a singularity can be defined as follows: the endeffector of the parallel manipulator can pass through the singular positions without perturbation of motion if the wrench applied on the end-effector by the legs, and external efforts of the manipulator are orthogonal to the twist along the direction of the uncontrollable motion. This condition is obtained from the inverse dynamics and analytically demonstrated by the study of the Lagrangian of a general parallel manipulator. Numerical simulations are carried out using the software ADAMS and validated by experimental tests.
\end{abstract}

Index Terms - parallel manipulators, singularity, controllability, dynamics.

\section{INTRODUCTION}

$\mathrm{P}$ arallel manipulators have experienced an increase in popularity in recent years due to their higher rate of acceleration, payload to weight ratio, stiffness and low effective inertia compared to those of serial manipulators. However they have some drawbacks, like a small workspace and special singular zones in it. Thus, in the presence of singular positions, the workspace of the parallel manipulators, which is less than that of serial manipulators, becomes even smaller and limits their functional performance. The studies of singularity have had different stages of development. The previous work on this problem is reported in a great number of publications and can be classified by different criteria. We arrange them in three major groups, distinguished by study of the singularity from kinematic, kinetostatic and dynamic points of view.

The physical interpretation of a singularity in kinematics refers to those configurations of parallel manipulators in which the number of degrees of freedom of the mechanical structure changes instantaneously, either the manipulator gains some additional, uncontrollable degrees of freedom or

Manuscript received September 12, 2007.

S. Briot and V. Arakelian are with the National Institute of Applied Sciences (I.N.S.A.), Rennes, 35043 France (corresponding author: +33223238492; fax: +33223238726; e-mail: vigen.arakelyan@insarennes.fr). loses some degrees of freedom. In this case the singularity analysis can be carried out on the base of the properties of the Jacobian matrices of the mechanical structure (i.e. when the Jacobian matrices relating the input speeds and the output speeds become rank deficient [1-4]), by using Grassmann geometry [5] or screw theory [6, 7].

However, it was observed that close to a singular configuration, a parallel manipulator loses its stiffness and its quality of motion transmission, and as a result, its payload capability. Thus, there are not only positions but rather singularity zones which must be avoided; and an indicator of the quality of motion transmission close to the singular configurations of parallel manipulators must be defined. For this purpose, a kinetostatic approach has been applied for the evaluation of the quality of motion transmission.

The quality of motion transmission of parallel manipulators was successfully studied in [8-11]. The quality of motion of manipulators with three degrees of freedom has been evaluated by means of a kinetostatic indicator, which is similar to the pressure angle [12]. In [13], the pressure angle was used as an indicator of the quality of motion transmission and the nature of the inaccessibility of singular zones by parallel manipulators are shown.

The study of singularities in parallel manipulators has revealed an interesting problem that concerns the path planning of parallel manipulators under the presence of singular positions, i.e. the motion feasibility in the neighborhood of singularities. In this case the dynamic conditions can be considered in the design process.

One of the most evident solutions for the stable motion generation in the neighborhood of singularities is to use redundant sensors and actuators [14-17]. However, it is an expensive solution to the problem because of the additional actuators and the complicated control of the manipulator caused by actuation redundancy. Another approach concerns with motion planning to pass through singularity [18-25], i.e. a parallel manipulator may track a path through singular poses if its velocity and acceleration are properly constrained. This is a promising path for the solution of this problem. However only a few research papers on this approach have addressed the path planning for obtaining a good tracking performance but they have not adequately addressed the physical interpretation of dynamic aspects.

In this paper, for the first time, the condition for passing through the singular positions is defined in general. It can be formulated as follows: "In the presence of a type 2 singularity, the platform of the parallel manipulator can pass through the singular positions without perturbation of 
motion if the wrench applied on the platform by the legs and external forces is orthogonal to the direction of uncontrollable motion". In other terms, the condition is that the work of applied forces and moments on the platform along the uncontrollable motion is equal to zero. This condition is obtained from the inverse dynamics and analytically demonstrated by the study of the Lagrangian of a general parallel manipulator. The obtained results are illustrated by numerical simulations and validated by experimental tests.

The paper is organized as follows. The next section presents theoretical aspects of the examined problem. Based on the Lagrangian formulation, the condition of force distribution is defined, that allows the passing of any parallel manipulator through the type 2 singular positions. In Section 3 , an application of the obtained theoretical results is examined for a parallel manipulator with 4 degrees of freedom. In section 4 , the numerical simulations carried out using the software ADAMS are validated by experimental tests

\section{OPTIMAL DYNAMIC CONDITIONS FOR PASSING THROUGH TYPE 2 SINGULARITY}

Let us consider a parallel manipulator of $m$ links, $n$ degrees of freedom and driven by $n$ actuators.

The Lagrangian dynamic formulation for a parallel manipulator can be expressed as:

$$
\tau=\frac{\mathrm{d}}{\mathrm{dt}}\left(\frac{\partial L}{\partial \dot{\mathbf{q}}}\right)-\frac{\partial L}{\partial \mathbf{q}}+\mathbf{B}^{T} \boldsymbol{\lambda},
$$

where,

- $\tau$ is the vector of the input efforts;

- $L$ is the Lagrangian of the manipulator;

- $\mathbf{q}=\left[q_{1}, q_{2}, \ldots, q_{n}\right]^{T}$ and $\dot{\mathbf{q}}=\left[\dot{q}_{1}, \dot{q}_{2}, \ldots, \dot{q}_{n}\right]^{T}$ represent the vector of active joints variables and the active joints velocities, respectively;

- $\mathbf{x}=[x, y, z, \phi, \psi, \theta]^{T} \quad$ and $\quad \mathbf{v}=[\dot{x}, \dot{y}, \dot{z}, \dot{\phi}, \dot{\psi}, \dot{\theta}]^{T} \quad$ are trajectory parameters and their derivatives, respectively; $x$, $y, z$ represent the position of the controlled point and $\phi, \psi$ and $\theta$ the rotation of the platform about three independent axes $\mathbf{a}_{\phi}, \mathbf{a}_{\psi}$ and $\mathbf{a}_{\theta}$ );

- $\lambda$ is the Lagrange multipliers vector, which is related to the wrench applied on the platform by:

$$
\lambda=\mathbf{A}^{-T} \mathbf{W}_{\mathbf{p}}
$$

where,

- A and $\mathbf{B}$ are two matrices relating the vectors $\mathbf{v}$ and $\dot{\mathbf{q}}$ according to $\mathbf{A v}=\mathbf{B} \dot{\mathbf{q}}$. They can be found by the closure equations with respect to time.

- $\mathbf{W}_{\mathbf{p}}$ is the wrench applied on the platform by the legs and external forces, which is defined as:

$$
\mathbf{W}_{\mathbf{p}}=\left(\frac{\mathrm{d}}{\mathrm{dt}}\left(\frac{\partial L}{\partial \mathbf{v}}\right)-\frac{\partial L}{\partial \mathbf{x}}\right)=\left[\begin{array}{l}
\mathbf{f}_{\mathbf{p}} \\
\mathbf{n}_{\mathbf{p}}
\end{array}\right] .
$$

where $\mathbf{f}_{\mathbf{p}}$ is the force along the directions of the global frame and $\mathbf{n}_{\mathbf{p}}$ is the torque about the axes $\mathbf{a}_{\phi}, \mathbf{a}_{\psi}$ and $\mathbf{a}_{\theta}$.
The term $\mathbf{W}_{\mathbf{p}}$ can be rewritten in the base frame using a transformation matrix $\mathbf{D}[26]$ :

$$
\mathbf{W}_{\mathbf{p}}=\mathbf{D}\left({ }^{\mathbf{R}_{0}} \mathbf{W}_{\mathbf{p}}\right)
$$

with ${ }^{\mathbf{R}_{0}} \mathbf{W}_{\mathbf{p}}$ is the expression of the wrench $\mathbf{W}_{\mathbf{p}}$ in the base frame, and

$$
\mathbf{D}=\left[\begin{array}{ll}
\mathbf{I}_{3 \times 3} & \mathbf{0}_{3 \times 3} \\
\mathbf{0}_{3 \times 3} & \mathbf{R}_{3 \times 3}
\end{array}\right]
$$

where $\mathbf{I}_{3 \times 3}, \mathbf{0}_{3 \times 3}$ and $\mathbf{R}_{3 \times 3}$ are respectively the identity matrix, the zero matrix and the transformation matrix between axes $\mathbf{a}_{\phi}, \mathbf{a}_{\psi}$ and $\mathbf{a}_{\theta}$ and the base frame, which dimensions are $3 \times 3$.

By substituting (5) into (1), one can obtain:

$$
\tau=\mathbf{W}_{\mathbf{b}}+\mathbf{J}^{T \mathbf{R}_{0}} \mathbf{W}_{\mathbf{p}}, \mathbf{W}_{\mathbf{b}}=\frac{\mathrm{d}}{\mathrm{dt}}\left(\frac{\partial L}{\partial \dot{\mathbf{q}}}\right)-\frac{\partial L}{\partial \mathbf{q}}
$$

where $\mathbf{J}=\left({ }^{\mathbf{R}_{0}} \mathbf{A}\right)^{-1} \mathbf{B}$ is the Jacobian matrix between twist $\mathbf{t}$ of the platform (expressed in the base frame) and $\dot{\mathbf{q}}$, ${ }^{\mathbf{R}_{0}} \mathbf{A}=\mathbf{A} \mathbf{D}$ is the expression of matrix $\mathbf{A}$ in the base frame.

For any prescribed trajectory $\mathbf{x}(t)$, the values of vectors $\ddot{\mathbf{q}}, \dot{\mathbf{q}}$ and $\mathbf{q}$ can be found using the inverse kinematics. Thus, taking into account that the manipulator is not in a type 1 singularity [1], the terms $\mathbf{W}_{\mathbf{b}}$ and ${ }^{\mathbf{R}_{0}} \mathbf{W}_{\mathbf{p}}$ can be computed.

However, for a trajectory passing through a type 2 singularity, the determinant of matrix $\mathbf{J}$ tends to infinity. Numerically, the values of the efforts applied by the actuators become infinite. In practice, the manipulator either is locked in such a position of the end-effector or it generates an uncontrolled motion. That is the end-effector of the manipulator produces a motion, different to the prescribed trajectory.

It is known that a type 2 singularity appears when the determinant of matrix ${ }^{\mathbf{R}_{0}} \mathbf{A}$ vanishes, in other words, when at least two of its columns are linearly dependant [26].

Let us rewrite the matrix ${ }^{\mathbf{R}_{0}} \mathbf{A}$ as:

$$
{ }^{\mathbf{R}_{0}} \mathbf{A}=\left[\begin{array}{cccc}
a_{11} & a_{12} & \ldots & a_{16} \\
a_{21} & a_{22} & \ldots & a_{26} \\
\vdots & \vdots & \ddots & \vdots \\
a_{61} & a_{62} & \ldots & a_{66}
\end{array}\right]=\left[\begin{array}{c}
\mathbf{R}_{1} \\
\mathbf{R}_{2} \\
\vdots \\
\mathbf{R}_{6}
\end{array}\right]
$$

In this expression, vector $\mathbf{R}_{i}=\left[a_{i 1}, a_{i 2}, \ldots, a_{i 6}\right](i=1, \ldots$, 6) represents the direction of the wrench applied on the platform by the $i$-th actuator of the mechanism [26].

If the columns of matrix ${ }^{\mathbf{R}_{0}} \mathbf{A}$ are linearly dependant, we can write:

$$
\sum_{j=1}^{6} \alpha_{j} R_{i j}=0, i=1, \ldots, 6
$$

where $R_{i j}$ is the $j$-th component of the vector $\mathbf{R}_{i}$ and $\alpha_{j}$ are the coefficients, which in general can be functions of $q_{p}, p=$ $1, \ldots, n)$. Thus, it is possible to show that the vector $\mathbf{t}_{\mathbf{s}}=$ $\left[\alpha_{1}, \alpha_{2}, \ldots, \alpha_{6}\right]^{T}$ is the reciprocal twist to the wrenches $\mathbf{R}_{\boldsymbol{i}}$. This vector represents the direction of the free motion of the platform in a type 2 singularity. 
Rewriting (8) in a vector form, we obtain:

$$
\sum_{j=1}^{6} \alpha_{j} \mathbf{N}_{j}=\mathbf{0}, \mathbf{N}_{j}=\left[a_{1 j}, a_{2 j}, \ldots, a_{n j}\right]^{T}, j=1, \ldots, 6
$$

where $\mathbf{N}_{j}$ represents the $j$-th column of matrix ${ }^{\mathbf{R}_{0}} \mathbf{A}$.

By substituting (9) into (2), we have

$$
\mathbf{N}_{j}^{T} \lambda=W_{j}, j=1, \ldots, 6
$$

where $W_{j}$ is the $j$-th row of vector ${ }^{\mathbf{R}_{0}} \mathbf{W}_{\mathbf{p}}$.

Then, from (9) and (10) the following conditions are derived:

$$
\sum_{j=1}^{6}\left(\alpha_{j} \mathbf{N}_{j}^{T} \boldsymbol{\lambda}\right)=\sum_{j=1}^{6}\left(\alpha_{j} W_{j}\right)=0
$$

The right term of eq.(11) corresponds to the scalar product of vectors $\mathbf{t}_{\mathbf{s}}$ and ${ }^{\mathbf{R}_{0}} \mathbf{W}_{\mathbf{p}}$.

Thus, in the presence of a type 2 singularity, it is possible to satisfy conditions (11) if the wrench applied on the platform by the legs and external efforts are orthogonal to the direction of the uncontrollable motion (singular motion). Otherwise, the dynamic model is not consistent. Obviously, in the presence of a type 2 singularity, the displacement of the end-effector of the manipulator has to be planned to satisfy (11). In the case of an inconsistent trajectory close to or through the singular positions, the required input efforts gain importance and a feasible motion is unattainable.

\section{ILLUSTRATIVE EXAMPLE}

In this section, the above discussed theory is illustrated by the example of a spatial parallel manipulator, PAMINSA, developed in the I.N.S.A. of Rennes, France [27]. Then, the results of numerical simulations carried out by the software ADAMS are tested on the built prototype.

The particularity of the PAMINSA, is in decoupling of the displacements of the platform in the horizontal plane from the translations along the vertical axis. The advantages of such an approach was discussed in [28, 29] and the singularity analysis is discussed in $[30,31]$.

The previous studies have revealed that there are type 2 singularities in the workspace of the symmetrical architecture of PAMINSA. In this section, we study the possibility of passing through the singular positions by this manipulator.

Fig. 1 shows the PAMINSA with four degrees of freedom. Each leg of the manipulator is realized by a pantograph mechanism (Fig. 2) with two input points $3_{i}$ and $8_{i}$, and an output point $5_{i}(i=1,2,3)$. Each input point $8_{i}$ is connected to the rotating drive $M_{i}$ by means of a prismatic guide mounted on a rotating link. This kind of architecture allows for generation of motion in the horizontal plane by the use of rotating actuators $M_{1}, M_{2}, M_{3}$, and the vertical translations by means of the linear actuator $M_{v}$. Thus, the displacements $(x, y, \phi)$ of the platform in the horizontal plane $\boldsymbol{x} \boldsymbol{O} \boldsymbol{y}$, that are translations along the $\boldsymbol{x}$ and $\boldsymbol{y}$-axes and rotations about the $\boldsymbol{z}$-axis, are independent of vertical

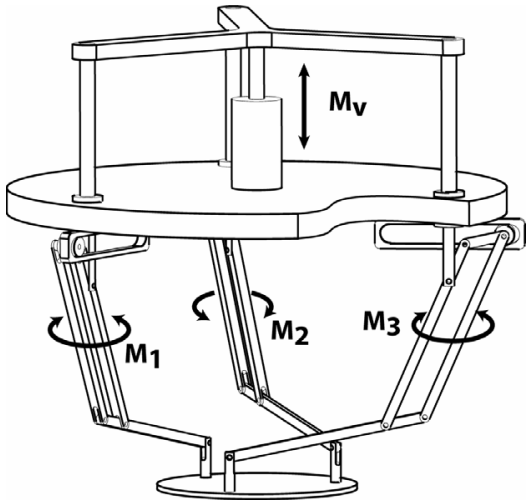

Fig. 1. PAMINSA: Parallel Manipulator of the I.N.S.A. [27].

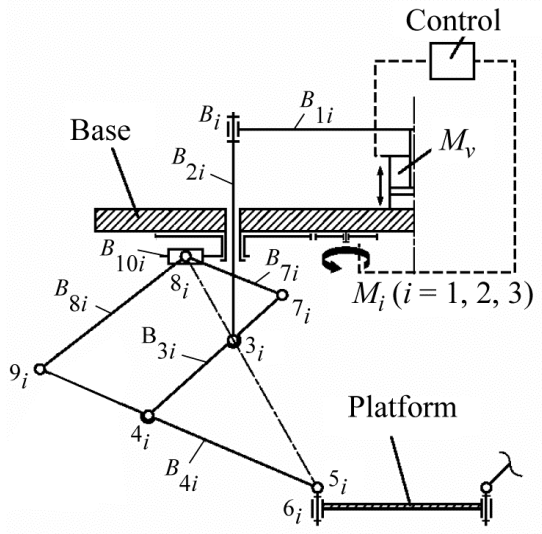

Fig. 2. Kinematic chain of each leg.

translations $z$. This implies that the kinematic models controlling the displacement of the manipulator can be divided into two parts:

- a model for the displacements in the horizontal plane, which is equivalent to a 3-RPR manipulator;

- a model for the translations along the vertical axis equivalent to the model for the vertical translations of a pantograph linkage.

The type 2 singularities of such a manipulator appear when [30, 31]:

a) the three legs of the manipulators are parallel, which is impossible for the developed PAMINSA manipulator.

b) the orientation of the platform is equal to $\cos ^{-1}\left(R_{p l} / R_{b}\right)$, where $R_{p l}$ and $R_{b}$ correspond respectively to the lengths $P C_{i}$ and $O M_{i}^{\prime}$ (Fig. 3). In this case, the manipulator gains one infinitesimal rotation around one vertical axis.

c) the platform is located in a circle defined by

$$
x^{2}+y^{2}=R_{p l}^{2}+R_{b}^{2}-2 R_{p l} R_{b} \cos \phi
$$

In this case, the manipulator gains one finite rotation about one vertical axis (Cardanic self motion) [32].

For cases (b) and (c), the direction of the unconstrained motion can be represented by the twist $\mathbf{t}_{\mathbf{s}}=\left[0,0,1, x_{W}, y_{W}\right.$, $0]^{T}$, where $x_{W}$ and $y_{W}$ correspond to the planar coordinates of the intersection point of the wrenches $\mathbf{R}_{i}$ applied on the platform by three legs of the manipulator (Fig. 3). 


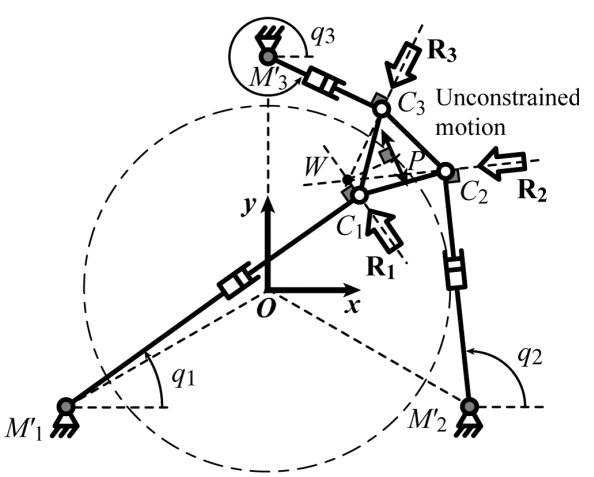

Fig. 3. Type 2 singular configuration of examined manipulator (horizontal projection of the examined structure).

Let us now study the inverse dynamics of the PAMINSA. The potential energy $V$ can be written as:

$$
V=V_{p l}+\sum_{i=1}^{3} V_{l g_{i}}
$$

where $V_{p l}$ is the potential energy of the platform and $V_{\text {leg }}$ is the potential energy of the leg $i(i=1,2,3)$.

By further considering that the coordinates of the all points of the pantograph linkages can be found as a linear combination of the coordinates of points $3_{i}, 5_{i}$ and $9_{i}$, one can express the terms $V_{p l}$ and $V_{\operatorname{leg}_{i}}$ as follows:

$$
\begin{gathered}
V_{p l}=m_{p l} g z \\
V_{\operatorname{leg}_{i}}=C_{v 1} z_{5 i}+C_{v 2} z_{9 i}+C_{v 3} q_{v}+C_{v 4}
\end{gathered}
$$

Here, $C_{v j}(j=1,2,3)$ are constant terms whose dimension is equivalent to a mass multiplied by the gravitational acceleration $g, m_{p l}$ is the mass of the platform with a payload, and $z_{5 i}$ and $z_{9 i}$ are the altitude of joints $5_{i}$ and $9_{i}$. The expressions of the coordinates of joints $5_{i}$ and $9_{i}$, as well as the expressions for $C_{v j}$ are complex, and their inclusion into this paper under the limited allowed length is not possible. An interested reader is referred to [33].

We consider that the links are perfect tubes. Therefore the tensor of inertia $\mathbf{I}_{j}$ of the link $B_{j i}$ at the center of masses will be written as:

$$
\mathbf{I}_{j}=\left[\begin{array}{ccc}
I_{X X}^{(j)} & 0 & 0 \\
0 & I_{Y Y}^{(j)} & 0 \\
0 & 0 & I_{Z Z}^{(j)}
\end{array}\right] \text {, with } I_{Y Y}^{(j)}=I_{Z Z}^{(j)}
$$

Thus, the kinetic energy $T$ of the manipulator can be represented as:

$$
T=T_{p l}+\sum_{i=1}^{3} T_{\operatorname{leg}_{i}},
$$

where $T_{p l}$ is the kinetic energy of the platform, $T_{\text {legi }}$ is the kinetic energy of the leg $i$, as

$$
T_{p l}=\frac{1}{2}\left(m_{p l}\left(\dot{x}^{2}+\dot{y}^{2}+\dot{z}^{2}\right)+I_{p l} \dot{\phi}^{2}\right)
$$

where $I_{p l}$ is the axial moment of inertia of the platform about the vertical axis.

$$
T_{\text {leg }_{i}}=T_{\text {trans }_{i}}+T_{\text {rot }_{i}}
$$

$$
\begin{aligned}
T_{\text {trans }_{i}}= & C_{c 1}\left(\dot{x}_{5 i}^{2}+\dot{y}_{5 i}^{2}\right)+C_{c 2} \dot{z}_{5 i}^{2}+C_{c 3}\left(\dot{x}_{9 i}^{2}+\dot{y}_{9 i}^{2}+\dot{z}_{9 i}^{2}\right) \\
& +C_{c 4}\left(\dot{x}_{5 i} \dot{x}_{9 i}+\dot{y}_{5 i} \dot{y}_{9 i}\right)+C_{c 5} \dot{z}_{5 i} \dot{z}_{9 i}+C_{c 6} \dot{q}_{v} \\
& +C_{c 7} \dot{z}_{5 i} \dot{q}_{v}+C_{c 8} \dot{q}_{i}^{2}
\end{aligned}
$$

$T_{r^{\prime} t_{i}}$ is the kinetic energy of the rotating links.

Note that there are two types of rotations (see, Fig. 1):

- rotation due to the actuators $M_{i}(i=1,2,3)$ (angle $q_{i}$ ), which is about the vertical axis,

- rotations due to the displacement of the pantograph in the linkage plane (angles $\zeta_{i}$ and $\varepsilon_{i}$ denoted as the angles between the direction of the passive slider and the links $B_{4 i}$ and $B_{3 i}$ respectively).

Thus, the kinetic energy of the rotating links can be written as:

$$
\begin{aligned}
T_{\text {rot }_{i}} & =C_{c 9} \dot{\zeta}_{i}^{2}+C_{c 10} \dot{\varepsilon}_{i}^{2}+\dot{q}_{i}^{2}\left(C_{c 13}+C_{c 10} \sin ^{2} \varsigma_{i}\right. \\
& \left.+C_{c 9} \cos ^{2} \varsigma_{i}+C_{c 12} \sin ^{2} \varepsilon_{i}+C_{c 11} \cos ^{2} \varepsilon_{i}\right)
\end{aligned}
$$

The expressions for $C_{c j}(j=1, \ldots, 13)$ are given in [33].

The input torques can be obtained from (6):

$$
\tau=\mathbf{W}_{\mathbf{b}}+\mathbf{J}_{P A M}^{T} \mathbf{W}_{\mathbf{p}}
$$

where the terms $\mathbf{J}_{P A M}, \mathbf{W}_{\mathbf{b}}$ and $\mathbf{W}_{\mathbf{p}}$ are presented in [30].

The following parameters of manipulator's links are specified for the trajectory generation:

- the radii of the circles circumscribed to the base and platform triangles are respectively equal to $R_{b}=0.35 \mathrm{~m}$ and $R_{p l}=0.1 \mathrm{~m}$;

- magnification factor of the pantograph: $k=3$;

- gravitational acceleration $g$ is equal to $9.81 \mathrm{~m} / \mathrm{s}^{2}$.

- lengths of the links of the pantograph linkages: $L_{B 1}=$ $0.308 \mathrm{~m}, L_{B 2}=0.442 \mathrm{~m}, L_{B 3}=L_{B 8}=0.42 \mathrm{~m}, L_{B 4}=k L_{B 7}=$ $0.63 \mathrm{~m}, L_{B 5}=0.0275 \mathrm{~m}, L_{B 10}=0.3635 \mathrm{~m}$;

- masses of the joints of the pantograph linkages: $m_{2}=$ $0.214 \mathrm{~kg}, m_{3}=0.338 \mathrm{~kg}, m_{4}=0.262 \mathrm{~kg}, m_{5}=0.233 \mathrm{~kg}$, $m_{7}=3.08 \mathrm{~kg}, m_{8}=0.305 \mathrm{~kg}, m_{9}=0.259 \mathrm{~kg}$;

- mass of the platform: $m_{p l}=2.301 \mathrm{~kg}$;

- masses of the links of the pantograph linkages: $m_{B 1}$ $=1.221 \mathrm{~kg}, m_{B 2}=0.921 \mathrm{~kg}, m_{B 3}=0.406 \mathrm{~kg}, m_{B 4}=0.672$ $\mathrm{kg}, m_{B 7}=0.107 \mathrm{~kg}, m_{B 8}=0.403 \mathrm{~kg}, m_{B 10}=0.436 \mathrm{~kg}$;

- term of the inertia matrix of the platform: $I_{p l}=0.015 \mathrm{~kg} / \mathrm{m}^{2}$.

- terms of the inertia matrices of the links of the pantograph linkages:

$$
\begin{aligned}
& I_{X X}^{(B 3)}=0.0038 \mathrm{~kg} / \mathrm{m}^{2}, I_{Y Y}^{(B 33)}=0.02 \mathrm{~kg} / \mathrm{m}^{2}, \\
& I_{X X}^{(B 4)}=0.0012 \mathrm{~kg} / \mathrm{m}^{2}, I_{Y Y}^{(B 4)}=0.048 \mathrm{~kg} / \mathrm{m}^{2}, \\
& I_{X X}^{(B 7)}=8 \cdot 10^{-4} \mathrm{~kg} / \mathrm{m}^{2}, I_{Y Y}^{(B 7)}=0.003 \mathrm{~kg} / \mathrm{m}^{2}, \\
& I_{X X}^{(B 8)}=0.0024 \mathrm{~kg} / \mathrm{m}^{2}, I_{Y Y}^{(B 8)}=0.02 \mathrm{~kg} / \mathrm{m}^{2}, \\
& I_{B 2}=0.003 \mathrm{~kg} / \mathrm{m}^{2}, I_{B 10}=0.02 \mathrm{~kg} / \mathrm{m}^{2} .
\end{aligned}
$$

The point $P$ is desired to make a motion $x(t)$ along a straight line between points $P_{0}\left(x_{0}, y_{0}\right)=P_{0}(0,0)$ and point $P_{f}\left(x_{f}, y_{f}\right)=P_{f}(0.3,0)$ in $t_{f}=2.4 \mathrm{~s}$. However, the manipulator will pass through a type 2 singular position at point $P_{s}\left(x_{s}\right.$, $\left.y_{s}\right)=(0.25,0)($ Fig. 4) 


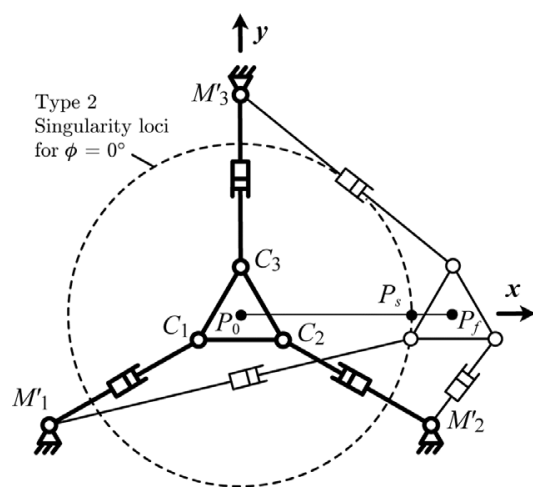

Fig. 4. Displacement of the PAMINSA along the prescribed straight line divided into two parts.

In order to carry out a comparative analysis for the optimized and not optimized dynamic conditions for passing through type 2 singularity, it has been considered two cases. The first is such a movement on the given trajectory, which is calculated from condition (11), and the second is an arbitrary motion.

At first let us consider an optimized trajectory which allows satisfying the condition (11), i.e. the force $\mathrm{W}_{\mathrm{p}}$ should be perpendicular to the to the twist $\mathrm{t}_{\mathrm{s}}[0,0,1,0,0.1,0]^{T}$ defining the direction of the unconstrained motion. Developing the expression (11) for the PAMINSA at point $P_{s}$, we obtain:

$$
\begin{aligned}
0 & =0.06441 \ddot{x}+1.2115 \ddot{y}-0.14649 \ddot{z}+0.04425 \ddot{\phi}+0.06827 \\
& +6.85084 \dot{x}^{2}+0.11720 \dot{y}^{2}-0.18482 \dot{z}^{2}+0.02947 \dot{\phi}^{2} \\
& -0.85175 \dot{\phi} \dot{x}+0.05643 \dot{\phi} \dot{y}+0.19423 \dot{\phi} \dot{z}-5.17625 \dot{x} \dot{y} \\
& +0.46477 \dot{x} \dot{z}+2.94694 \dot{y} \dot{z}
\end{aligned}
$$

Now considering that the end-effector of the manipulator moves along a straight line directed along the $\boldsymbol{x}$-axis, we can note that $\dot{y}\left(t_{s}\right)=\dot{z}\left(t_{s}\right)=\ddot{y}\left(t_{s}\right)=\ddot{z}\left(t_{s}\right)=\dot{\phi}\left(t_{s}\right)=$ $\ddot{\phi}\left(t_{s}\right)=0$. Thus, the relationships, which satisfy the passing through of the singular positions, taking into account that the velocity and the acceleration of the platform in the initial and final positions are equal to zero, can be expressed by the following boundary conditions:

$$
\begin{gathered}
x\left(t_{0}\right)=x_{0}, \\
x\left(t_{f}\right)=x_{f}, \\
x\left(t_{s}=2 \mathrm{~s}\right)=x_{s}, \\
\dot{x}\left(t_{0}\right)=0, \\
\dot{x}\left(t_{f}\right)=0, \\
\ddot{x}\left(t_{0}\right)=0, \\
\dot{x}\left(t_{s}\right)=\dot{x}_{s}=0.05 \mathrm{~m} / \mathrm{s}, \\
\ddot{x}\left(t_{s}\right)=\ddot{x}_{s}=-1.32583 \mathrm{~m} / \mathrm{s}^{2} .
\end{gathered}
$$

In this case, a motion for passing of the platform through the singular position can be found from the following eighth order polynomial form:

$$
\begin{aligned}
x(t) & =3.41 t^{8}-37.65 t^{7}+166.05 t^{6}-365.23 t^{5} \\
& +400.63 t^{4}-175.27 t^{3}
\end{aligned}
$$

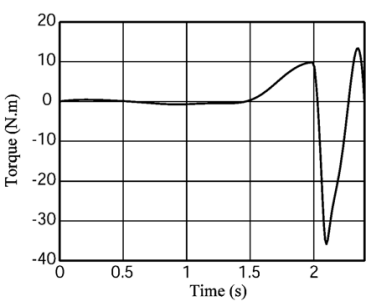

(a) actuator $M_{1}$.

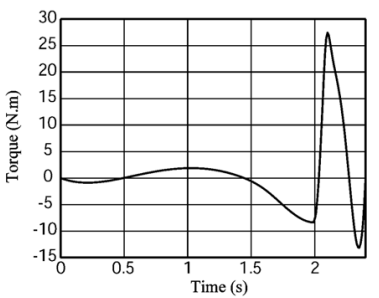

(c) actuator $M_{3}$.

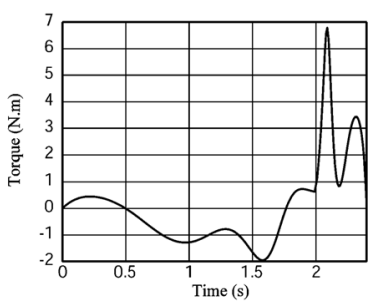

(b) actuator $M_{2}$.

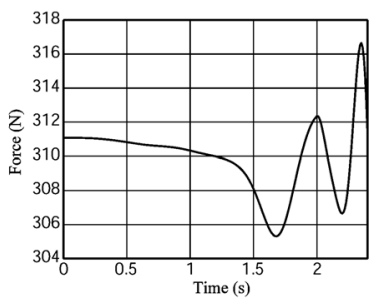

(d) actuator $M_{v}$.
Fig. 5. Input efforts of the PAMINSA in the case of the sixth order polynomial trajectory planning, obtained by the ADAMS software.

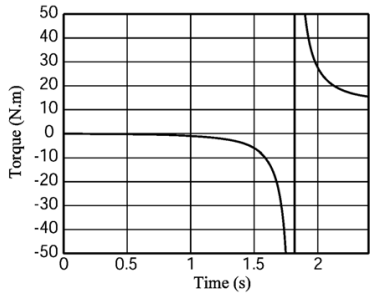

(a) actuator $M_{1}$.

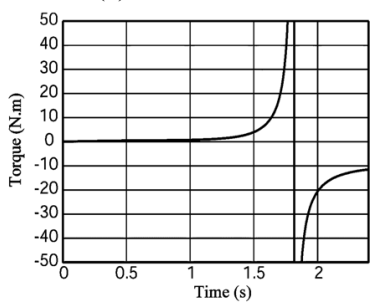

(c) actuator $M_{3}$

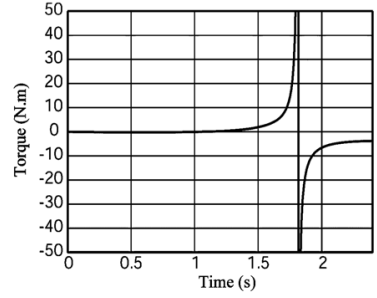

(b) actuator $M_{2}$.

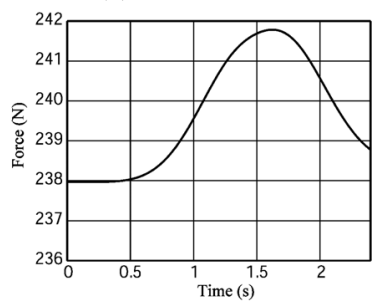

(d) actuator $M_{v}$.
Fig. 6. Input efforts of the PAMINSA in the case of the fifth order polynomial trajectory planning obtained by the software ADAMS.

However, a trajectory obtained by (36) cannot be reproduced by the prototype because of the limited capability of drivers' deceleration. Therefore, the trajectory was divided into two parts, i.e., the first sixth order polynomial trajectory assumes the motion from an initial to the singular position $\left(P_{0} P_{s}\right)$ and the second sixth order polynomial trajectory- from singular to the final position $\left(P_{s} P_{f}\right)$. The core of the problem is same but it allows for generating motions for the prototype.

Thus, the trajectory planning equations can be written as:

$$
\begin{aligned}
x(t)= & x_{0}+\left(x_{s}-x_{0}\right)\left(b_{3} t^{3}+b_{4} t^{4}+b_{5} t^{5}+b_{6} t^{6}\right) \text { for } \mathrm{t} \leq t_{s} ;(37) \\
x(t)= & x_{s}+\left(x_{f}-x_{s}\right)\left(c_{1}\left(t_{f}-t_{s}\right)+c_{2}\left(t_{f}-t_{s}\right)^{2}+c_{4}\left(t_{f}-t_{s}\right)^{4}\right. \\
& \left.+c_{5}\left(t_{f}-t_{s}\right)^{5}+c_{6}\left(t_{f}-t_{s}\right)^{6}\right)
\end{aligned}
$$

for $t>t_{s}$. 
with $b_{3}=-3.3033, b_{4}=5.10456, b_{5}=-2.45207, b_{6}=0.37844$, $c_{1}=1, c_{2}=-13.25829, c_{4}=2365.3672, c_{5}=-11953.07236$ and $c_{6}=16158.76157$.

Thus, the motion obtained from sixth order polynomial equations allows for passing through the singularity without perturbation, and the input efforts take on finite values (Fig. 5).

It can be seen that by the end of the motion there is an increase of the input efforts, caused by a quick deceleration to stop the manipulator before it reaches the workspace boundary.

It will be shown further that in the case of the motion generated by any trajectory planning without meeting the adopted boundary conditions (27) - (35), the manipulator platform is not able to pass through the singular position. For demonstration purpose, the generation of motion between initial and final positions is carried out by a fifth order polynomial trajectory planning.

In this case, for $y(t)=0 \mathrm{~m}, z(t)=-0.45 \mathrm{~m}$ and $\phi(t)=0^{\circ}$, the fifth order polynomial trajectory planning is the following:

$x(t)=x_{0}+\left(x_{f}-x_{0}\right)\left(10\left(\frac{t}{t_{f}}\right)^{3}-15\left(\frac{t}{t_{f}}\right)^{4}+6\left(\frac{t}{t_{f}}\right)^{5}\right)$

The obtained input efforts are represented in Fig. 6.

It can be noted that for arbitrary trajectory while the manipulator passes through the singular configuration (for $t_{s}$ $\approx 1.8 \mathrm{~s}$ ), the value of the input torques tend to infinity.

\section{EXPERIMENTAL VALIDATION OF OBTAINED RESULTS}

For validating the results of the previous section, we have carried out experimental tests on the prototype of the PAMINSA with 4 DOF developed in the I.N.S.A. of Rennes (Fig. 7).

We have applied an arbitrary fifth order control law and observed the reproduction of motion during the displacement of the platform. The obtained trajectory is shown in Fig. 8 (dotted line).

The different positions are classified by time. For positions from (a) to (d), the platform moves towards the singular zone but yet it is outside of it. In this case, the reproduction of the real trajectory is similar to the desirable. At position (e), the manipulator enters the singular zone, which is close to the circle of the theoretical singular loci, and starts an uncontrollable motion. Thus, since the motion generation is carried out by non optimized dynamic parameters, the platform moves along an unplanned trajectory (see positions (e) - (h) in Fig. 8).

Next, we have implemented the sixth order control laws as it was shown in the previous section and observed the behavior of the platform during the displacement. The different positions are classified by time. During all these displacements, the manipulator retains its orientation and passes through the singular configuration without any perturbation.

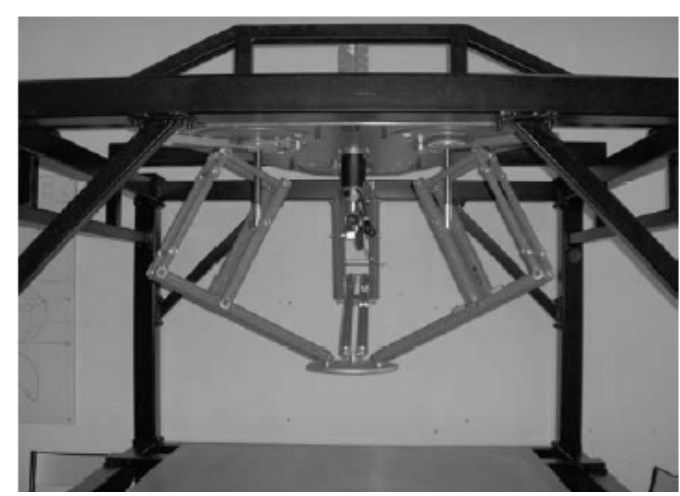

Fig. 7. The built prototype of the PAMINSA with 4 DOF, which was used for the experimental validation of obtained results.
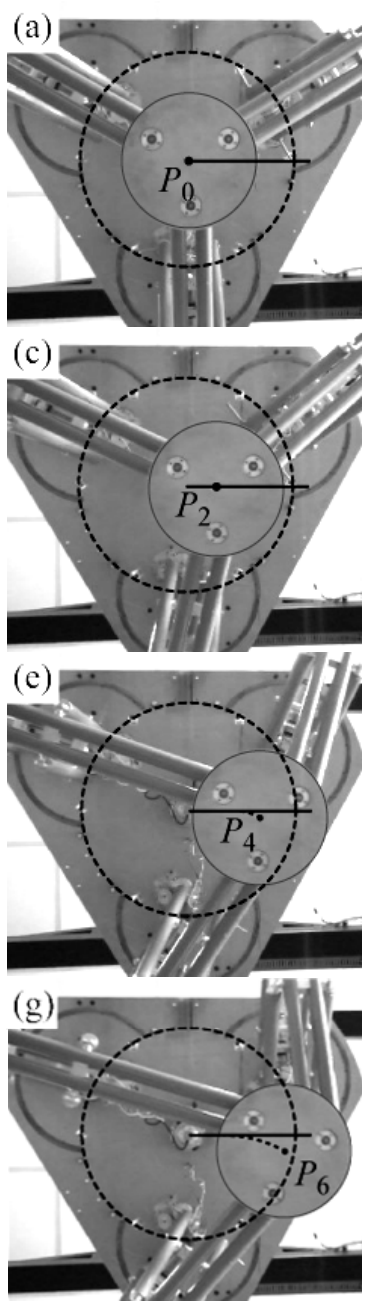
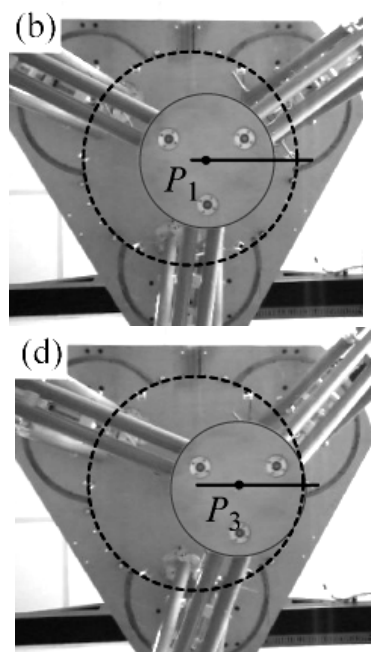

(f)
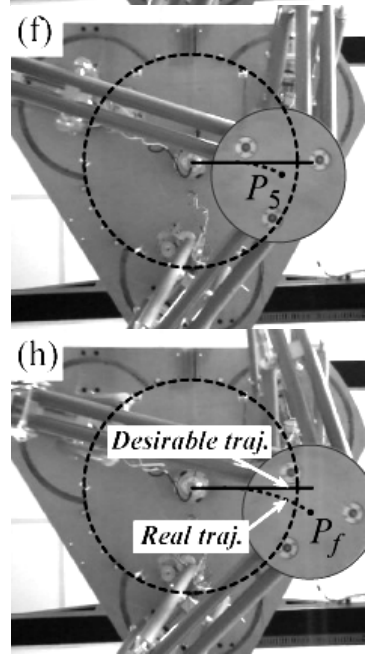

Fig. 8. Trajectory reproduction on the PAMINSA during the displacement of the platform with the fifth order polynomial law (view from below).

\section{CONCLUSION}

The singularities limit the functional performances of parallel manipulators. The best known solutions to increase the size of the workspace of parallel mechanisms, based on the actuation redundancy, are expensive and cannot be widely adopted. Therefore, other means for passing through singular configurations need to be developed. 
In this paper we have found the optimal dynamic conditions, for making the pass through the type 2 singular configurations possible. The general definition of the condition for passing through the singular position is formulated as follows: in the presence of type 2 singular configuration, the platform of a parallel manipulator can pass through the singular positions without perturbation of motion if the wrench applied on the platform by the legs and external efforts are orthogonal to the direction of the uncontrollable motion, or in other words, if the work of applied forces and moments on the platform along the uncontrollable motion is equal to zero. This condition has been verified by numerical simulations carried out with the software ADAMS and validated by experimental tests on the prototype of four degrees of freedom parallel manipulator PAMINSA.

We point out that for the case of non controllable external forces applied on the platform the proposed technique cannot be used. Therefore, the most prominent field of the industrial application is a "fast pick and place" manipulation, when the generation of motion is determined by input, gravitational and inertia forces.

\section{REFERENCES}

[1] C.M. Gosselin, and J. Angeles, "Singularity analysis of closed-loop kinematic chains," IEEE Transactions on Robotics and Automatics, Vol. 6, No. 3, 1990, pp.281-290.

[2] O. Ma, and J. Angeles, "Architecture singularities of parallel manipulators," The International Journal of Robotics and Automation, Vol. 7, No. 1, 1992, pp.23-29.

[3] E. Ottaviano, C.M. Gosselin, and M. Ceccarelli, "Singularity analysis of CaPaMan: a three-degree of freedom spatial parallel manipulator," in Proc. 2001 IEEE International Conference on Robotics and Automation, Seoul, Korea, May 21-26, 2001, pp. 1295-1300.

[4] J.T. Wen, J.F. Oapos Brien. Singularities in three-legged platformtype parallel mechanisms. IEEE Transactions on Robotics and Automation. 19(4) (2003) pp.720- 726.

[5] J.-P. Merlet, "Singular configurations of parallel manipulators and Grassmann geometry," The International Journal of Robotics Research, Vol. 8, No. 5, 1989, pp. 45-56.

[6] K.H. Hunt, "Special configurations of robot-arms via screw theory," Robotica, Vol. 5, 1987, pp. 17-22.

[7] I.A. Bonev, D. Zlatanov, and C.M. Gosselin, "Singularity analysis of 3-DOF planar parallel mechanisms via Screw Theory," Transactions of the ASME. Journal of Mechanical Design, Vol. 125, 2003, pp. 573581.

[8] H.S. Kim and Y.J. Choi, "Forward/inverse force transmission capability analyses of fully parallel manipulators," EEE Transactions on Robotics and Automation, 2001, 17 (4), pp. 526-531.

[9] S.-H. Lee, J.-B. Song, W.C. Choi, D. Hong, "Workspace and forcemoment transmission of a variable arm type parallel manipulator," Proceedings - IEEE International Conference on Robotics and Automation, 2002, 4, pp. 3666-3671.

[10] S.-G. Kim and J. Ryu, "Force transmission analyses with dimensionally homogeneous jacobian matrices for parallel manipulators," KSME International Journal, 2004, 18 (5), pp. 780788.

[11] S. Weiwei and C. Shuang, "Study on force transmission performance for planar parallel manipulator," Proceedings of the World Congress on Intelligent Control and Automation (WCICA), 2006, 2, art. no. 1713551, pp. 8098-8102.

[12] O. Alba-Gomez, P. Wenger, and A. Pamanes, "Consistent kinetostatic indices for planar 3-DOF parallel manipulators, application to the optimal kinematic inversion," in Proc. ASME 2005 IDETC/CIE Conference, Long Beach, California, September 24-28, 2005.
[13] V. Arakelian, S. Briot, and V. Glazunov, "Increase of singularity-free zones in the workspace of parallel manipulators using mechanisms of variable structure," Mechanism and Machine Theory, in press.

[14] C.L. Collins, and G.L. Long, "The singularity analysis of an parallel hand controller for force-reflected teleoperation," IEEE Transactions on Robotic and Automation, Vol. 11, No. 5, 1975, pp. 661-669.

[15] B. Dasgupta, and T. Mruthyunjaya, "Force redundancy in parallel manipulators: theoretical and practical issues," Mechanism and Machine Theory, Vol. 33, No. 6, 1998, pp.724-742.

[16] V. Glazunov, A. Kraynev, R. Bykov, G. Rashoyan, and N. Novikova, "Parallel manipulator control while intersecting singular zones," in Proc. 15th Symposium on Theory and Practice of Robots and Manipulators (RoManSy) CISM-IFToMM, Montreal, 2004.

[17] K. Alvan, and A. Slousch, "On the control of the spatial parallel manipulators with several degrees of freedom," Mechanism and Machine Theory, Saint-Petersburg, No. 1, 2003, pp. 63-69.

[18] S. Bhattacharya, H. Hatwal, and A. Ghosh, "Comparison of an exact and approximate methode of singularity avoidance in platform type parallel manipulators," Mechanism and Machine Theory, Vol. 33, No. 7, 1998, pp. 965-974.

[19] B. Dasgupta, and T. Mruthyunjaya, "Singularity-free path planning for the Steward platform manipulator," Mechanism and Machine Theory, Vol. 33, No. 6, 1998, pp. 715-725.

[20] M.H. Perng, and L. Hsiao, "Inverse kinematic solutions for a fully parallel robot with singularity robustness," The International Journal of Robotics Research, Vol. 18, No. 6, 1999, pp. 575-583.

[21] S. Kemal Ider, "Inverse dynamics of parallel manipulators in the presence of drive singularities," Mechanism and Machine Theory, Vol. 40, 2005, pp. 33-44.

[22] C.K. Kevin Jui, and Qiao Sun, "Path tracking of parallel manipulators in the presence of force singularity," Transactions of the ASME. Journal of Dynamic Systems, Measurement and Control, Vol. 127, December, 2005, pp. 550-563.

[23] A.K. Dash, I.-M. Chen, S.H. Yeo, and G. Yang, "Workspace generation and planning singularity-free path for parallel manipulators," Mechanism and Machine Theory, Vol. 40, No. 7, 2005, pp. $778-805$.

[24] D.N. Nenchev, and M. Uchiyama, "Singularity-consistent path planning and control of parallel robot motion through instantaneousself-motion," IEEE Int. Conf. on Robotics and Automation, Minneapolis, USA, 1996, pp. 1864-1870.

[25] D.N. Nenchev, S. Bhattacharya, and M. Uchiyama, "Dynamic analysis of parallel manipulator under the singularity-consistent parameterization," Robotica, Vol. 4, No. 4, 1997, pp. 375-384.

[26] J.-P Merlet, "Parallel Robots," Springer, 2nd edition, 2006.

[27] V. Arakelian, P. Maurine, S. Briot, and E. Pion, "Parallel robot comprising means for setting in motion a mobile element split in two separate subassemblies," Patent FR2873317 (A1), WO 2006/021629, January 27, 2006.

[28] V. Arakelian, S. Briot, S. Guegan, and J. Le Flecher, "Design and prototyping of new 4-, 5- and 6-DOF parallel manipulators based on the copying properties of the pantograph linkage," in Proc. 36th International Symposium on Robotics, Tokyo, Japan, November 29 December 1, 2005.

[29] V. Arakelian, S. Briot, and V. Glazunov, "Singular positions of a PAMINSA parallel manipulator," Journal of Machinery Manufacture and Reliability, Allerton Press Inc., No 1, 2006, pp. 62-69.

[30] S. Briot, S. Guegan, E. Courteille, and V. Arakelian, "On the Design of PAMINSA: a New Class of Parallel Manipulators with High-Load Carrying Capacity," in Proc. ASME 2007 IDETC/CIE Conference, Las Vegas, Nevada, USA, September 4-7, 2007.

[31] S. Briot, and V. Arakelian, "Singularity analysis of PAMINSA manipulators," in Proc. 12th World Congress in Mechanism and Machine Science, Besançon, France, June 18-21, 2007.

[32] D. Chablat, P. Wenger, and I.A. Bonev, "Self motions of a special 3RPR planar parallel robot," in Advances in robot kinematics, J. Lenarcic and B. Roth (eds.), Springer, 2006, pp. 221-228.

[33] S. Briot, "Analysis and optimization of a new family of parallel manipulators with decoupled motions," $P h D$. Thesis, 2007. 